\title{
Aproximaciones al lóbulo frontal. Una mirada hacia la rehabilitación
}

\author{
María Rocío Acosta Barreto*
}

\section{Resumen}

La región prefrontal es la estructura más directamente relacionada con el funcionamiento ejecutivo, cognoscitivo y conductual. Es una estructura que es especialmente vulnerable a lesiones cerebrales inducidas como traumas craneoencefálicos, accidentes cerebrovasculares, tumores, trastornos neurodegenerativos y por trastornos del neurodesarrollo. Éstas dejan como secuelas alteración en la capacidad de inhibición, fallas en programación, déficit en memoria operativa y ordenamiento temporal, cambios en conducta social, pseudodepresión, apatía, pérdida de la motivación e iniciativa y dificultad para la inhibición de sus respuestas. La intervención en esta población genera retos para los profesionales de salud quienes han de mantener habilidades personales y profesionales que proyecten en el paciente confianza y efectiva adhesión al tratamiento.

Palabras clave: función ejecutiva, conducta ejecutiva, síndrome prefrontal, rehabilitación frontal.

\section{Approaches to frontal lobes. A look toward the rehabilitation}

\begin{abstract}
The prefrontal region is the most directly structure related to executive functioning, cognitive and behavioral. Is a structure that is particularly vulnerable to brain damage induced as cephalic trauma, cerebrovascular accidents, tumors, neurodegenerative disorders and neurodevelopmental disorders. These make alterations as sequelae of inhibition in the ability, faulty programming, and working memory deficits in temporal order, changes in social behavior, pseudodepression, apathy, loss of motivation and initiative and difficulty inhibiting their responses. The intervention in this population creates challenges for health professionals who maintain personal and professional skills that project in the patient the trust and effective adherence to treatment.
\end{abstract}

Key word: executive function, executive behavior, prefrontal syndrome, front rehabilitation. 


\section{Introducción}

De acuerdo con el último estudio neuroepidemiológico nacional colombiano publicado en la Revista Panamericana de Salud Públi$c a$ en el año 2002, en donde se entrevistó a 8.910 personas residentes de 2.560 viviendas (1.303 personas en la región oriental; 3.077 en la central; 1.591 en la noroccidental; 998 en la suroccidental; y 1.941 en la costa Caribe), se encontró a 57 personas con secuelas de trauma craneoencefálico respecto del total, con una prevalencia de 6,4 por 1.000 habitantes en un intervalo de confianza del 95\% (5.0 a 7.8). (Pradilla, Vesga, León-Sarmiento y Grupo GENECO, 2003).

Esta prevalencia, que si bien es cierto fue considerada en el estudio como la séptima causa de enfermedad neurológica, es de gran magnitud y merece especial atención por los altos costos que implica no sólo en términos de la limitación que genera para la reincorporación a la vida familiar, laboral y social de quien la padece, sino por manejo intrahospitalario.

De hecho muchos de estos pacientes deben ser pensionados a corta edad y generan intervenciones médicas no sólo por servicios de Neurología sino por Fisiatría, Terapias Física, Ocupacional y del Lenguaje, Psicología, Neuropsicología y Psiquiatría, incrementando aun más el costo de la atención en salud por paciente. A esto se suma que muchos quedan con secuelas que alteran su conducta social con lo que se acentúa la probabilidad de incrementar costos en medicamentos para inhibir sus impulsos y modular el afecto, dada la dificultad de la familia y del paciente mismo para controlarse, generar un compromiso de cambio y modificar su conducta.

Aunque se ha referido en los dos párrafos anteriores al trauma craneoencefálico, una situación igual se evidencia en otras problemáticas como los accidentes cerebrovasculares, los tumores y los trastornos neurodegenerativos y del desarrollo, todos éstos dejan igualmente un importante grado de discapacidad no sólo física sino cognoscitiva y conductual. Esto implica un importante reto de abordaje a disfunción secundaria de los lóbulos frontales.

\section{Definición}

La corteza prefrontal es la estructura filogenéticamente más reciente y ontogenéticamente una de las últimas en alcanzar su completa mielenización, (Casey y col., 1997) se encuentra relacionada con funciones de alta complejidad al ser ésta la gerente de funciones mentales superiores y por ende del comportamiento, sirviendo de filtro, planificador, verificador o control y rector de los procesos a los cuales se les ha denominado función y conducta ejecutiva. (Anderson, Anderson, Northam, Jacobs \& Mikiewicz, 2002).

Ardila (2008) plantea:

"Las funciones ejecutivas se han convertido en el elemento clave para la comprensión de la racionalidad y la conducta social humana. Generalmente, suponemos que al menos algunos de los componentes de las funciones ejecutivas sólo se encuentran en la especie humana, y en consecuencia, las funciones ejecutivas pueden ser la clave para comprender la cultura, la civilización, la ciencia y la tecnología".

La función ejecutiva ha sido definida por diferentes autores entre los que se cuentan Lezak quien en 1989 expresa: "comprende las capacidades mentales necesarias para formular metas, planificar la manera de lograrla y llevar adelante ese plan de manera eficaz". Sholberg y Mateer en este mismo año señalan: "La función ejecutiva abarca una serie de procesos cognitivos entre los que destacan la anticipación, la elección de objetivos, la planificación, la selección de la conducta, la autorregulación, el autocontrol y el uso de retroalimentación". Barkley en 2001 por su parte dijo: "es un modelo de acción autodirigido que permite la maximización global de las consecuencias sociales, una vez que las consecuencias inmediatas y demoradas de las distintas alternativas de acción se consideran simultáneamente" (Bausela y Santos, 2004). 
Slachevsky y cols. (2005) en su trabajo sobre los modelos explicativos de la función ejecutiva, recogen desde el punto de vista anatómico y funcional cinco modelos, los cuales se describen a continuación.

Modelo Luria (1980): En este modelo se destaca que es la tercera unidad funcional y sus neuronas terciarias tienen el papel de programar, regular y verificar la conducta. El comportamiento se da en cuatro etapas: análisis de los datos iniciales, elaboración de un programa que organiza y ordena las diferentes actividades necesarias para la realización de la tarea, ejecución del programa y confrontación del resultado con los datos iniciales.

Modelo Shalice (1982): Constituido por un repertorio de acciones motrices e intelectuales habituales cuya ejecución relativamente automática permite enfrentar las situaciones repetitivas de la vida cotidiana y un sistema de supervisión atencional que interviene cuando una actividad nueva o compleja necesita la elaboración de estrategias.

Modelo Fuster (1986): la corteza prefrontal permite la integración temporal del comportamiento, mediante la intervención de la memoria de trabajo, la inhibición y la preparación para el logro de metas.
Modelo Mesulam (2002): La corteza prefrontal suprime y trasciende respuestas automáticas e inflexibles a unas respuestas más flexibles y contingentes. Y se manifiesta a través de las siguientes funciones: memoria de trabajo; inhibición de la distractibilidad, de la perseveración y de la satisfacción inmediata; búsqueda activa de la novedad; significación emocional; y codificación del contexto, toma de perspectiva y comprensión de los otros.

Modelo Koechlin y cols. (2003): En esta propuesta se describe la organización antero-posterior de la corteza prefrontal lateral en el control cognitivo. El modelo distingue cuatros niveles de control de la acción: el control sensorial, asociado al córtex premotor e implicado en la selección de acciones motoras en respuestas a estímulos; las regiones caudales de la corteza prefrontal lateral (áreas 9, 44 y 45 de Brodmann), implicadas en el control contextual; las regiones rostrales de la misma corteza, relacionadas con el control episódico; y las regiones polares (área 10 de Brodmann), implicadas en el control de las ramificaciones, es decir, en la activación de las representaciones prefrontales rostrales (Slachevsky, Pérez, Silva, Orellana, Prenafeta, Alegria y Peña, 2005).

TABla 1.

RESUMEN AUTORES FUNCIÓN EJECUTIVA

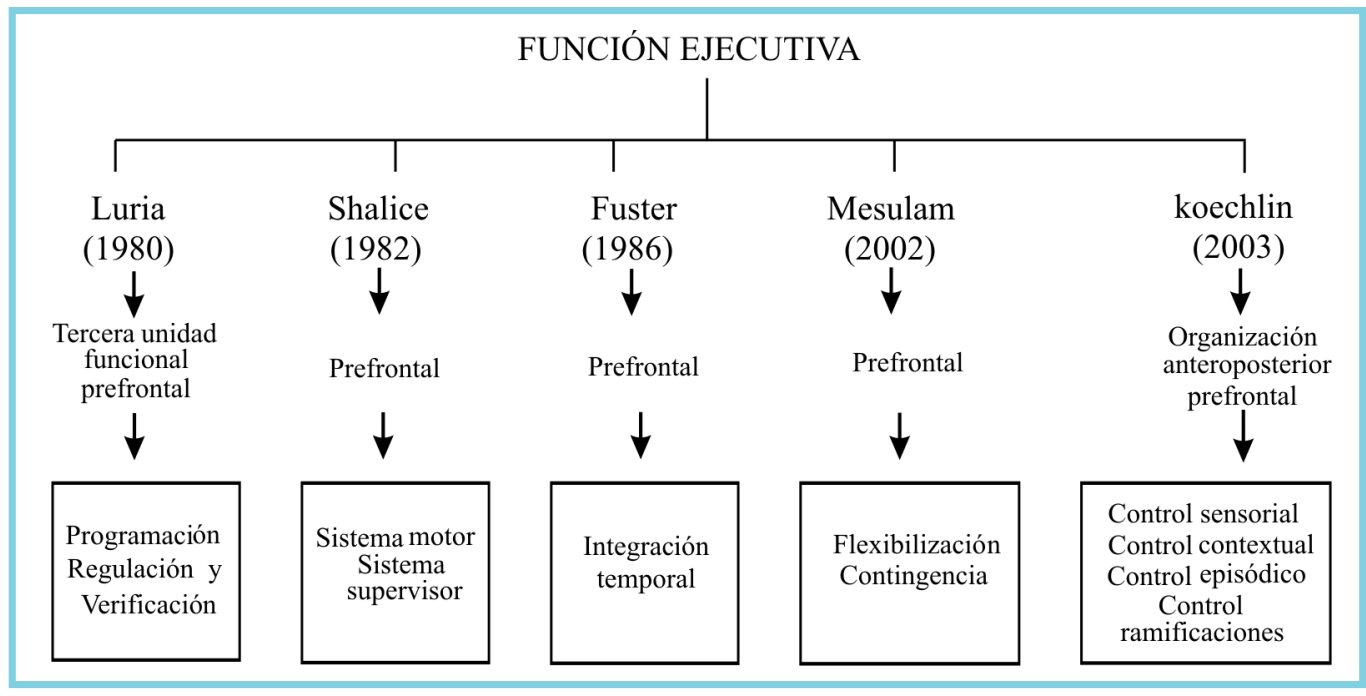


De otro lado, se encuentra la conducta ejecutiva que hace referencia al control y regulación de la conducta y se define bajo los siguientes modelos:

Modelo de Stuss y Benson (1986): Es un modelo neuroanatómico conductual por el que se entiende que el lóbulo frontal regula la conducta a través de tres niveles de procesamiento básico, los que poseen una estructura jerárquica:

- En la base el nivel sensoperceptual que se caracteriza por ser de alta velocidad, de procesamiento automático, rutinario y rígido al cambio y no requiere de un procesamiento consciente.

- El otro nivel es el de control ejecutivo en el que se requiere de un procesamiento controlado, es menos veloz y exige altos recursos atencionales y cognoscitivos; se encarga de anticipar, planear, implementar y monitorear el comportamiento y se implica con la atención selectiva y sostenida.

- El último nivel en la cima, es el de autoconciencia y metacognición que está relacionado con el funcionamiento de regiones prefrontales y se caracteriza por un manejo consciente, regulado y autorreflexivo que involucra la inversión de altos recursos atencionales y cognoscitivos implicados con nuestra conducta. (Stuss, Gallup y Alexander, 2001).

Modelo de Kauffer y Lewis (1992): En algunos textos citado como modelo de Cummings: este modelo habla de tres tipos de corteza, la dorsolateral, la orbitofrontal y la ventromedial.
La corteza dorsolateral (áreas 9 y 10 de Brodmann) actúa como una memoria a corto plazo que permite elegir entre varias opciones posibles y aprender de los propios errores. A estaárea Mesulam, (citado por Jòrdar-Vicente, 2004) agrega que una de las funciones principales de esta región es la de propiciar la interacción inicial entre la información sensorial que recibe del córtex posterior y la información procedente del sistema límbico y el córtex paralímbico. Esta interacción implica la relación existente y la retroalimentación entre las sensaciones y el humor: la forma en que las emociones influyen en la interpretación de la información sensorial y la forma en que el procesamiento y los aprendizajes previos pueden modificar los estados de ánimo.

La corteza orbitrofrontal (área 47, 11, 13 y 14 de Brodman y giro frontal inferior) se encuentra situada en la parte inferior de los lóbulos prefrontales y encima de los ojos y tiene la capacidad de llevar a la práctica la opción elegida y ha sido relacionada con el control inhibitorio, el control emocional y el comportamiento.

Finalmente, la corteza ventromedial recibe proyecciones de las mismas zonas de la corteza orbitofrontal pero con la diferencia de recibir la mayor cantidad de proyecciones provenientes de zonas hipocámpicas y sensoriales auditivas. Esta región se relaciona con el control emocional inhibitorio permitiendo el cambio de conducta según el significado emocional de los estímulos y está relacionada con la comunicación emocional y la intencionalidad para la acción. (Sánchez-Navarro y Román, 2004).

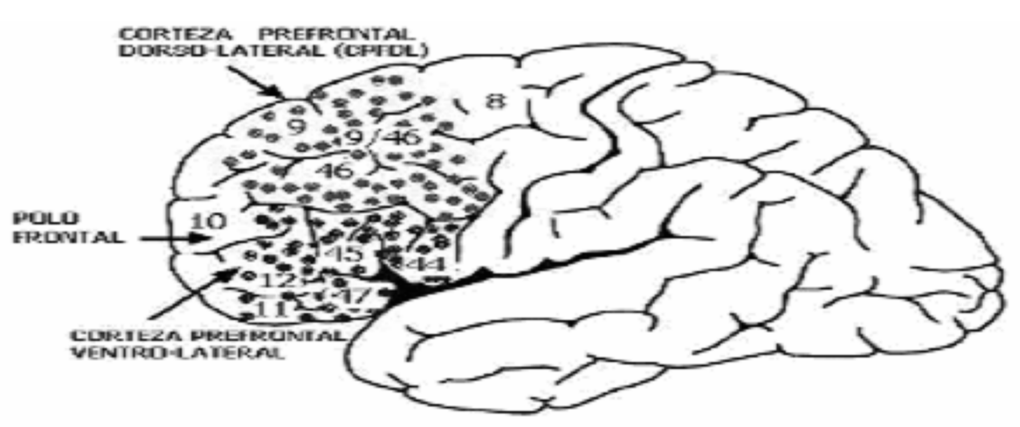

www.revmed.unal.edu.co/.../v54n4/v54n4a05.html 
Modelo de Damasio: Este autor muestra la implicación que tiene la vida emocional y motivacional en el surgimiento de la conciencia. El autor plantea la hipótesis del marcador somático por el cual, cuando se ha de tomar una decisión se experimenta en el cuerpo unas respuestas especificas. Así como las emociones (componente importante de los procesos cognitivos) son acompañadas de respuestas autonómicas y de alerta previamente condicionadas por estímulos externos, éstas pueden servir como una señal o marcador (marcador somático) que guía la cognición; el aprendizaje y la toma de decisiones. Basándose en esto Damasio encuentra que lesiones de la corteza prefrontal ventral medial (incluida el área 25) impide que los pacientes utilicen estos marcadores somáticos para guiar la toma de decisiones. En cambio individuos normales manifiestan la respuesta autonómica anticipatoria y desarrollan una estrategia adecuada para tareas cognoscitivas. (Damasio, 1994).
Es así como los marcadores somáticos, se generan a través de aprendizaje asociativo, conectando emociones y sentimientos a resultados futuros predecibles. Este sentimiento "marca" la imagen representada con una tonalidad de la emoción correspondiente, y lo llama somático porque el sentimiento se produce en el cuerpo, es la reacción de éste lo que marca a la representación.

Los pacientes con daño en el lóbulo prefrontal evocan el conocimiento relacionado con contenidos de imágenes que se les muestran, pero no se da en ellos respuesta emocional, no funciona su marcador somático cuando evocan. Esto les conduce a un extraño síndrome, ya que aparentemente muestran normalidad, pero cuando se les trata o se conoce más sobre sus vidas se ve que no funcionan bien, ya que han perdido algo significativo y necesario para tomar decisiones, lo que hacen que acaben fracasando personal y profesionalmente. Damasio explicita en una frase lo anterior "saber no significa necesariamente sentir". (Damasio y Anderson, 1993).

\section{Tabla 2.}

\section{RESUMEN AUTORES CONDUCTA EJECUTIVA}

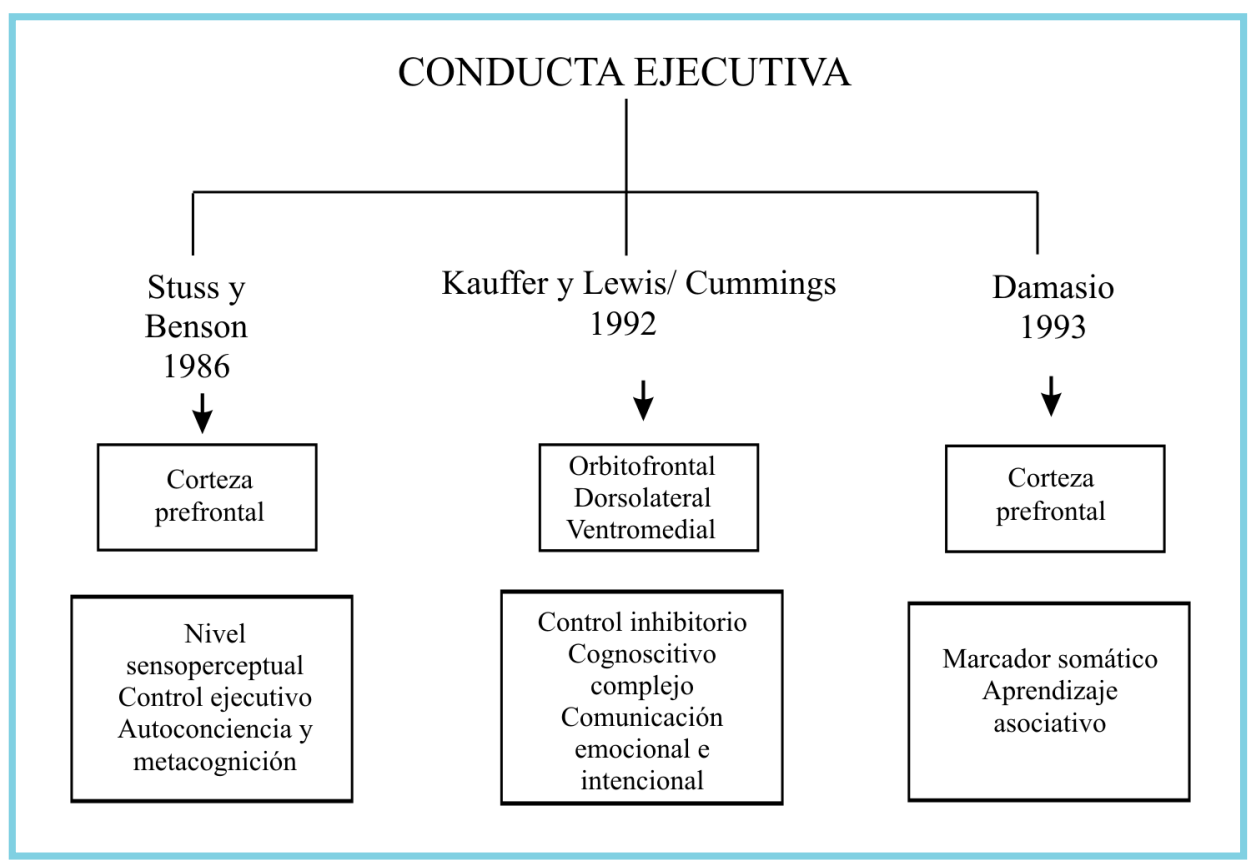


Otras aproximaciones llevan a considerar la división del lóbulo frontal en función de sus conexiones talámicas (Jòrdar-Vicente, 2004). Aquí, cada zona cortical recibe proyecciones de núcleos talámicos específicos: Córtex precentral: incluye área premotora y área motora suplementaria, y sus proyecciones proceden de los núcleos ventromediales. Córtex prefrontal o anterior: recibe proyecciones del núcleo dorsomedial del tálamo. Córtex cingular: con proyecciones que provienen del núcleo ventral anterior.

La representación jerárquica en la mediación del lóbulo frontal en la ejecución de las acciones incluye las neuronas motoras, los núcleos motores, el cerebelo, el tálamo, los ganglios basales y el córtex frontal. Al mismo tiempo, este último también se organizaría jerárquicamente: el córtex motor primario mediaría en la representación y ejecución de movimientos esqueléticos; el córtex premotor actuaría en la programación de los movimientos más complejos, que implican meta y trayectoria; y el córtex prefrontal, donde se produce la representación de mayor nivel, actuaría a través de la distribución de redes de neuronas cuya actividad puede verse 'limitada' por la coincidencia temporal de la actividad y el input a través de tres funciones cognitivas básicas: La memoria a corto plazo, la motora y la preparación para la acción, en el área motora suplementaria, la memoria perceptiva a corto plazo (memoria de trabajo) para la retención de la información sensorial relevante, en el córtex dorsolateral, el control inhibitorio de la interferencia para eliminar aquello que es irrelevante, en el córtex orbital. (Merlo, Albanese, Gómez, Miño, Ingrata, Mascitti y Albanese, 1999).

\section{Aspectos patológicos}

Frente a lesión cerebral se presentan secuelas a nivel de la función y la conducta ejecutiva en diferentes niveles, buena parte de ellos incapacitantes para la vida familiar y social y con limitaciones importantes para el proceso de rehabilitación funcional.
Tras lesiones prefrontales se evidencian variados déficit cognoscitivos a nivel de la memoria, reducción de los niveles de atención y concentración, problemas visoespaciales y visomanipulativos, pérdida de habilidades de razonamiento y una pobre planificación y capacidad de organización. Y adicionalmente, se presentan numerosos cambios emocionales y psicosociales como apatía, falta de iniciativa, irritabilidad y bajo control de la impulsividad, escasa tolerancia a la frustración, reducida conciencia de las limitaciones y pobres habilidades sociales. (Lezak, 1982).

Retomando autores como Damasio y Anderson (1993); Koechlin, Basso, Pietrini, Panzer y Grafman (1999), se resumen a continuación algunas de las secuelas dejadas por lesión frontal, a partir de las principales áreas prefrontales:

Ante lesiones orbitofrontales se han descrito individuos con incapacidad para llevar a cabo la opción seleccionada, sólo actúan a corto plazo tratando de satisfacer sus necesidades o deseos inmediatos. Se caracterizan por desinhibición, pérdida del control social del comportamiento, tendencia a explorar todo por el tacto y a reír sin razón aparente, les cuesta entender el sentido de los chistes, exhiben comportamiento eufórico y muestran algunos elementos obsesivo-compulsivos.

Lesiones dorsolaterales se han relacionado con alteración en función ejecutiva: limitación o ausencia en la capacidad de generar hipótesis, en la planeación y la toma de decisiones, en la focalización de la atención, en el análisis de sus resultados y en la inhibición de estímulos irrelevantes. Alteraciones en la memoria de trabajo, en la metamemoria, además de inefectividad en los mecanismos de codificación, pobre intensión y motivación para permanecer en la tarea, dificultad para el orden temporal de las acciones, reducción en fluidez verbal y no verbal, fallas en programación motora y alteraciones en la conducta como apatía, lentificación, inatención, desmotivación, distractibilidad, dependencia del ambiente, pérdida de curiosidad y en lesiones izquierdas, depresión. 
Diversas lesiones descritas en sujetos humanos y no humanos (monos) han dejado ver que las alteraciones en el control inhibitorio, se han encontrado tanto en lesiones orbitofrontales como dorsomediales, implicando una fuerte asociación de estas áreas con el comportamiento emocional y el comportamiento mediado por estímulos. (Roberts y Wallis, 2000).

Tras lesiones ventromediales sobresale mutismo acinético, total apatía y respuestas guiadas por motivos aparentes vs. Desmotivación total. Dificultades en la planificación de las actividades cotidianas, y en la toma de decisiones, alteraciones anímicas, disminución profunda de la competencia social, deterioro severo de la conducta sexual, pero las capacidades ejecutivas de resolución de problemas formales, de lenguaje y de memoria permanecen relativamente intactas. Administran su dinero de forma inconsciente, les es difícil mantener un empleo, tienen planes pobres con metas poco realistas, pueden emplear mucho tiempo tratando de resolver un conflicto trivial, como qué ropa vestir. Presentan gran vulnerabilidad al engaño y a sufrir abuso por otras personas. (Contreras, Catena, Candido, Perales y Maldonado, 2008).

\section{Rehabilitación neuropsicológica}

Sohlberg y Mateer (1989) definen a la rehabilitación neuropsicológica como un proceso terapéutico que involucra numerosas estrategias que tienen el objetivo de incrementar o mejorar la capacidad del individuo para procesar y usar la información de su ambiente, y para permitir inadecuado funcionamiento en la vida cotidiana.

Es así como la rehabilitación se ve como un proceso mediante el cual los pacientes con daño cerebral y los profesionales del servicio de salud, trabajan en conjunto para remediar o aliviar los déficits cognitivos que surgen tras una afección neurológica. (Wilson, 1991).

Existen, como se había mencionado anteriormente, numerosas posibilidades que implican las lesiones del lóbulo frontal. Y esto se agrava porque en su gran mayoría estos pacientes al ingresar para ser evaluados, no presentan secuelas en su capacidad motora ni discapacidades evidentes para su desempeño en la vida diaria. Adicionalmente, no muestran trastornos evidentes en su capacidad de lenguaje, por lo que estos pacientes, pueden no ser descubiertos aunque existan deterioros en la memoria, la visuoespacialidad o en el funcionamiento ejecutivo.

El deterioro del funcionamiento ejecutivo, es muy difícil de detectar, a menos que sea evaluado por un neuropsicólogo, o un especialista; muchas veces se hace evidente después de haber pasado mucho tiempo desde la lesión, solamente cuando las conductas inadecuadas y los cambios comportamentales han lesionado fuertemente la vida del paciente.

Una evaluación exhaustiva dá las bases para el diseño de programas de rehabilitación acordes a las limitaciones de los pacientes, ésta implica tanto la exploración de la sintomatología física y cognoscitiva, así como los cambios conductuales y emocionales que presenta la persona. Lo que implica que independientemente del propósito de la intervención de cada profesional, se debe incluir en el protocolo de evaluación, la indagación completa en todas las áreas de funcionamiento de la persona, a fin de llegar con mayor propiedad al diagnóstico, y a una más segura intervención y conducta a seguir en la junta evaluadora interdisciplinaria.

En el caso de los pacientes que sufren de un síndrome del lóbulo frontal es usual encontrar asociado a una sintomatología cognoscitiva, cambios en su comportamiento y emociones, que en muchos casos pueden llegar a interferir con los objetivos de la rehabilitación, lo cual muchas veces se traduce en el abandono del proceso terapéutico por la persona, o también se encuentra enlentecimiento en el cumplimiento de objetivos lo que produce un agotamiento del personal de la salud, que lo esté tratando.

Es necesario por tanto que en el equipo de rehabilitación esté presente la intervención psicológica 
y neuropsicológica, que de acuerdo con Arango y Parra (2008), trabaje enfocándose primordialmente en el control de los siguientes problemas:

1) la iniciación, secuenciación, regulación e inhibición del comportamiento;

2) la solución de problemas;

3) el razonamiento abstracto; $y$

4) las alteraciones de la autoconciencia de la enfermedad.

Cuando no son abordadas estas circunstancias, se afecta en grado notorio la interacción con el cuerpo médico, ya que la exhibición de conductas como la agresividad, la apatía, la inconstancia en las tareas asignadas, la rigidez y las dificultades para el seguimiento atencional e intencional, promueven barreras que terminan en el abandono uni o bilateral de las intervenciones terapéuticas, lo que termina empeorando las condiciones de estos pacientes; quienes además de todas las problemáticas descritas anteriormente, empiezan a experimentar el rechazo social lo que genera mayor frustración y de alguna forma refuerza la manifestación de conductas perturbadoras, dado que estos pacientes tienen una mirada más dirigida al locus de control externo.

Lo negativo que le acontece a un paciente con lesión prefrontal tiende a estar directamente relacionado con los inadecuados manejos por parte de los profesionales de salud, el sobreproteccionismo o falta de prontitud o beligerancia de la familia o por la insensibilidad social, es la mirada interpretativa que hace el paciente frontalizado, asumiendo un carácter de victimización que le lleva a mostrarse con mayor hostilidad u aislamiento como medidas de defensa o protección.

Esta alta rigidez cognoscitiva, falta de autorretroalimentación, pobre capacidad de solución de problemas y baja tolerancia a la frustración, son por tanto un reto para los profesionales que trabajen con estas personas, porque para lograr resultados efectivos con estos pacientes, se requiere del diseño de estrategias efectivas para lograr su adaptación a la sociedad, y que sean útiles para su comunidad.

A partir de la experiencia de varios años en el manejo de pacientes con lesión frontal, se nombran a grandes rasgos unas indicaciones que le pueden ser útiles para el abordaje terapéutico:

1. Mantenga el control de sus emociones frente a estos pacientes, recuerde que es usted el que está sano. Pelear con una persona frontalizada sólo lo llevarán a desgastarse emocionalmente y a perder el respeto y la continuidad terapéutica de su paciente.

2. Brinde información clara repitiendo cada vez que sea necesario la importancia de cada una de las actividades que usted le asigna.

3. Las instrucciones verbales y escritas siempre deben ser cortas y claras.

4. Proporcione un clima de empatía, pero con plena claridad de los límites que usted mismo demarca desde el principio. Evite siempre la familiaridad.

5. Cuando el paciente se equivoque hágaselo saber, pero siempre con argumentos que resalten el motivo de su equivocación. Si usted no lo hace el paciente no podrá comprender el porqué de su error.

6. Realice por lo menos una vez cada mes una sesión de trabajo grupal en la cual el paciente pueda relacionarse con otros con secuelas similares, esto favorece la generación de redes de apoyo, fundamentales en los procesos terapéuticos con esta clase de pacientes.

7. Involucre a la familia dentro del proceso para que entienda en qué consiste el síndrome prefrontal, reciba apoyo emocional y haga continuidad en casa al manejo del equipo de salud.

8. Conforme un equipo interdisciplinario en el cual se fijen los objetivos de intervención y se hagan las disposiciones y ajuste necesarios. Todos deben tener el mismo comportamiento 
frente al paciente para lograr resultados efectivos en el menor tiempo posible.

Dentro del trabajo específico, es importante la implementación unificada del equipo de rehabilitación, de estrategias conductuales; éstas deben ser diseñadas con suficiente claridad, donde se contemplen los objetivos de intervención y el establecimiento de reforzadores a este cumplimiento de objetivos. En algunas ocasiones las técnicas cognitivo conductuales pueden ser de gran ayuda para lograr la disposición de los pacientes en la colaboración del proceso terapéutico, garantizando su adherencia al tratamiento y por lo tanto, su mejoría.

Sin embargo, dada la complejidad de estos pacientes, es importante generar investigaciones que evalúen la efectividad de las diferentes estrategias y técnicas terapéuticas, lo que a futuro podrían propender por la conformación de protocolos unificados y validados de intervención efectiva para el síndrome prefrontal.

Recibido: agosto 2008

Aceptado: octubre 2008

\section{Bibliografía}

Anderson V, Anderson P, Northam E, Jacobs R \& Mikiewicz O. Relationships between cognitive and behavioral measures of executive function in children with brain disease. Child Neuropsychology, 2002; 8, 231-240.

Arango Tobón OE, Puerta IC, Pineda D. Estructura factorial de la función ejecutiva desde el dominio conductual. Revista Diversitas - Perspectivas. En: Psicología. 2008; 4, 63-77.

Arango JC \& Parra M. Rehabilitación de las funciones ejecutivas en caso de patología cerebral. En: Revista Neuropsicología, Neuropsiquiatría y Neurociencias. Abril 2008; 8 (1): 159-178.

Ardila A. Editorial. En: Revista Neuropsicología, Neuropsiquiatría y Neurociencias. Abril 2008; 8 (1): 159-178.
Bausela E \& Santos J. Disfunción ejecutiva: Sintomatología que acompaña a la lesión y/ o disfunción del lóbulo frontal. En: Avances en Salud Mental Relacional. Bilbao (España). 2004; 5 (2).

Casey BJ, Castellanos FX, Giedd JN et al. Implication of right frontostriatal circuitry in response inhibition and attention - deficit hyperactivity disorder. J Am Acad Child Adolesc. Psychiatry 1997; 36: 374-383.

Contreras D, Catena A, Candido A, Perales J y Maldonado A. Funciones de la corteza prefrontal ventromedial en la toma de decisiones emocionales. Internacional Journal of Clinical and Health Psicology. 2008; 8 (1): 285-313.

Damasio AR \& Anderson SW. The frontal lobes. Clinical Neuropsychology. Heilman KM, Valenstein E (eds.) New York, Oxford University Press. 1993.

Damasio AR. Descartes' error. Emotion, reason and the human brain. New York: Avon Books. 1994.

Jòrdar-Vicente, M. Funciones cognitivas del lóbulo frontal. En: Revista Española de Neurología 2004; 39: 179-182.

Koechlin E, Basso G, Pietrini P, Panzer S \& Grafman J. The role of the anterior prefrontal cortex in human cognition. En: Nature. 1999; 399: 148-151.

Lezak M. The problem of assessing executive functions. En: International Journal of Psychology. 1982; 17: 281-297.

Merlo A, Albanese A, Gómez E, Miño J, Ingrata A, Mascitti T. \& Albanese E. Los giros del lóbulo frontal: estudio post mortem. En: Revista Chilena de Anatomía. 1999; 17: 2.

Pradilla G, Vesga B, León-Sarmiento F. \& Grupo GENECO, (2002). Estudio neuroepidemiológico nacional (EPINEURO) colombiano. En: Revista Panamericana de Salud Pública. Washington (2003). 14: 2.

Roberts A \& Wallis J. 2002. Inhibitory control and affective processing in the prefrontal cortex: 
Neuropsychological studies in the common marmoset. En: Cerebral Cortex Mar; Oxford University Press. 2004; 10: 252-256.

Sánchez-Navarro, J. \& Román, F. Amígdala, corteza prefrontal y especialización hemisférica en la experiencia y expresión emocional. En: Anales de Psicología. ; 20 (2): 223-240.

Slachevsky A, Pérez C, Silva J, Orellana G, Prenafeta M, Alegria P \& Peña M. 2005. Córtex prefrontal y trastornos del comportamiento: modelos explicativos y métodos de evalua- ción. En: Revista Chilena de Neuropsiquiatría. Versión on-line. 1989; 43 (2): 109-121.

Sohlberg MKM. and Mateer CA. Introduction to cognitive rehabilitation: theory and practice. Guilford Press. New York.

Stuss D, Gallup G \& Alexander M. 2001; The frontal lobes are necessary for theory of mind. Brain, 2001; 124 (2): 279-286.

Wilson BA. 1991; Theory, assessment, and treatment in neuropsychological rehabilitation. Neuropsychology. 1991; 5 (4): 281-291. 